\title{
Ocorrência de Babesia spp. em cães utilizando a técnica de esfregaço sanguíneo
}

\author{
Occurrence of Babesia spp. in dogs using the blood smear technique \\ Ocurrencia de Babesia spp. en perros mediante la técnica de frotis de sangre
}

Recebido: 12/01/2021 | Revisado: 18/01/2021 | Aceito: 26/01/2021 | Publicado: 02/02/2021

Bruna Louise Adona Reis Pereira
ORCID: https://orcid.org/0000-0001-8056-6319
Universidade Federal de Mato Grosso, Brasil
E-mail: adonabruna @ gmail.com
Eveline da Cruz Boa Sorte
ORCID: https://orcid.org/0000-0002-2831-5325
Universidade Federal de Mato Grosso, Brasil
E-mail: evelinesorte@ hotmail.com
Jaqueline Camargo Borges
ORCID: https://orcid.org/0000-0003-3533-7618
Universidade Federal de Mato Grosso, Brasil
E-mail: jaquelineborges13@ hotmail.com
Adriane Jorge Mendonça
ORCID: https://orcid.org/0000-0002-9367-5028
Universidade Federal de Mato Grosso, Brasil
E-mail: adrianejorge.m@gmail.com

\section{Resumo}

A babesiose é uma zoonose causada por diferentes espécies de protozoários do gênero Babesia, que obrigatoriamente vivem no interior das células sanguíneas. Transimitida por carrapatos ixodídeos, ela pode acometer tanto animais domésticos, quanto silvestres. No Centro-Oeste, apesar de já existirem descrições da hemoparasitose, pouco se sabe referente à sua ocorrência, sendo a maioria dos estudos referentes à detecção por meio de diagnósticos moleculares. Apesar disso, o esfregaço sanguíneo mostra-se um método diagnóstico fundamental, principalmente, por ser um exame de triagem, viável, altamente conclusivo e de baixo custo. Este estudo objetivou enfatizar a importância do esfregaço sanguíneo como diagnóstico da enfermidade. Realizou-se um levantamento dos achados de Babesia spp. em esfregaços sanguíneos da rotina do HOVET - UFMT, durante o período de doze meses, bem como foi buscada a existência de um perfil hematológico, visando validar a suspeita mesmo em casos de baixa parasitemia. Foram processados 5.333 hemogramas caninos, destes, os que apresentaram o trofozoíto da Babesia spp. na citologia dos esfregaços foram submetidos à avaliação do resultado de seus exames. Foi constatado que mesmo em casos que a citologia resulte negativa, com o histórico clínico associado ao perfil hematológico é possível validar a suspeita da hemoparasitose. Verificou-se a ocorrência de $0,3 \%$ de babesiose em cães, sendo a maioria dos achados esporádicos. Os principais achados hematológicos foram anemia $(68,75 \%)$ normocítica normocrômica $(45,45 \%)$, trombocitopenia $(87,5 \%)$, leucopenia $(75 \%)$, eosinopenia $(68,75 \%)$, linfopenia $(87,5 \%)$ e monocitose $(18,75 \%)$.

Palavras-chave: Hematozoários; Patologia clínica; Hematologia; Babesiose.

\begin{abstract}
Babesiosis is a zoonosis caused by different species of Babesia protozoa, which live inside the blood cells. Transmitted by ixodid ticks, it can affect both domestic and wild animals. In the Central-West Region, although there are already descriptions of this hemoparasitosis, little is known about its occurrence, and most studies refer to its detection through molecular diagnostics. Despite this, blood smear is a fundamental diagnostic method, mainly because it is a viable, highly conclusive and low-cost screening test. This study aimed to emphasize the importance of blood smear as a diagnosis for the disease. It was collected data on Babesia spp. findings in blood smears from the HOVET - UFMT routine, during the period of twelve months, and also searched for the existence of a hematological profile, aiming to validate the suspicion even in cases of low parasitemia. 5.333 canine hemograms were processed, of these, those that presented the Babesia spp. trophozoite in the smear cytology, were submitted to an exam evaluation. It was found that even in cases where the cytology results negative, with the clinical history associated to the hematological profile, it is possible to validate the suspicion of the hemoparasitosis. It was found a $0,3 \%$ occurrence of babesiosis in dogs, being mostly sporadic findings. The main hematological findings were anemia $(68,75 \%)$ normocytic normochromic $(45,45 \%)$, thrombocytopenia $(87,5 \%)$, leukopenia $(75 \%)$, eosinopenia $(68,75 \%)$, lymphopenia $(87,5 \%)$ and monocytosis $(18,75 \%)$.
\end{abstract}

Keywords: Hematozoa; Clinical pathology; Hematology; Babesiosis. 


\section{Resumen}

La babesiosis es una zoonosis causada por diferentes especies de protozoos del género Babesia, que obligatoriamente viven dentro de las células sanguíneas. Transmitida por garrapatas ixódidas, puede afectar tanto a animales domésticos como salvajes. En la región Centro-Oeste, aunque ya existen descripciones de esta hemoparasitosis, poco se sabe sobre su ocurrencia, siendo la mayoría de estudios referidos a la detección mediante diagnóstico molecular. A pesar de esto, el frotis de sangre es un método diagnóstico fundamental, principalmente porque es una prueba de detección viable, altamente concluyente y de bajo costo. El propósito del estúdio fue enfatizar la importancia del frotis de sangre como diagnóstico de la enfermedad. Se realizó una encuesta de los hallazgos de Babesia spp. en los frotis sanguíneos de rutina del HOVET - UFMT, durante el período de doce meses, así como se buscó la existencia de un perfil hematológico, con el objetivo de validar la sospecha en casos de parasitemia baja. Se procesaron 5.333 hemogramas caninos, de estos, los que presentaron lo trofozoíto de la Babesia spp. en la citología de frotis, también se evaluaron sus exámenes. Se encontró que incluso en los casos en que la citología sea negativa, con la historia clínica asociada al perfil hematológico, es posible validar la sospecha de la hemoparasitosis. Se verificó la ocurrencia de $0,3 \%$ de babesiosis en perros, siendo la mayoría de los hallazgos esporádicos. Los principales hallazgos hematológicos fueron anemia $(68,75 \%)$ normocítica normocrómica $(45,45 \%)$, trombocitopenia $(87,5 \%)$, leucopenia $(75 \%)$, eosinopenia $(68,75 \%)$, linfopenia $(87,5 \%)$ y monocitosis $(18,75 \%)$.

Palabras clave: Hematozoos; Patologia clinica; Hematologia; Babesiosis.

\section{Introdução}

Nos últimos anos, a aquisição de animais de companhia tem aumentado significativamente e, com isso, nota-se também uma relação mais próxima entre eles e os homens. Consequentemente, é possível observar uma alta na ocorrência de zoonoses, o que gera preocupação, visto que são enfermidades que acometem tanto os humanos quantos os animais (Day, 2011).

A babesiose é uma enfermidade que acomete diversas espécies de animais, domésticos e selvagens, até mesmo o homem. No Brasil, o primeiro registro de caso de infecção humana ocorreu no Estado de Pernambuco (Alecrim et al., 1983), e, recentemente, Serra-Freire (2014) registrou um novo caso no Rio de Janeiro, entretanto, poucos casos foram encontrados na literatura.

Há quatro espécies conhecidas por causar a babesiose canina em todo o mundo, Babesia canis, Babesia vogeli, Babesia rossi e Babesia gibsoni. Estas causam uma doença febril e hemolítica, que é considerada endêmica no país (Brandão \& Hagiwara, 2002). Ela é transmitida por carrapatos ixodídeos, que funcionam como vetores mecânicos (Castro, 2020). A prevalência e distribuição geográfica da enfermidade estão relacionadas diretamente com a distribuição do carrapato vetor, ocorrendo, principalmente, nas regiões tropicais e subtropicais (Andereg \& Passos, 1999).

No Centro-Oeste, apesar de já existirem descrições da hemoparasitose (Duarte et al., 2008; Santos et al., 2018; Castro, 2020), ainda há poucos dados referentes à ocorrência da mesma. Ademais, a maioria dos estudos são relacionados à sua detecção por meio de diagnósticos moleculares, pela possibilidade de classificação das subespécies e alta sensibilidade.

Em contrapartida, o esfregaço sanguíneo mostra-se também um importante método diagnóstico, em detrimento de sua viabilidade, baixo custo, alta conclusividade, e, principalmente, por ser um exame de triagem, visto que esta hemoparasitose apresenta sinais clínicos altamente inespecíficos, dentre eles, anorexia, apatia, diarreia, febre, anemia branda a grave e icterícia (Vercammen, Deken \& Maes, 1995; Dias \& Ferreira, 2016).

Diante do exposto, devido à importância diagnóstica dessa enfermidade, este estudo objetivou realizar um levantamento dos achados de Babesia spp. em esfregaços sanguíneos da rotina do HOVET - UFMT, durante o período de doze meses, com a finalidade de evidenciar a importância deste método diagnóstico. Além disso, buscou-se analisar se há um perfil hematológico que possibilite validar a suspeita mesmo em casos de baixa parasitemia, visando incentivar a solicitação de exames de maior sensibilidade para confirmação nos casos em que a microscopia direta resulte negativo. 


\section{Metodologia}

O estudo retrospectivo foi realizado nas dependências do HOVET - UFMT, campus Cuiabá, recolhendo dados dos arquivos do Laboratório de Patologia Clínica Veterinária (LPCV), de exames realizados no período de janeiro a dezembro de 2019.

Durante o ano, foram realizados 5.333 hemogramas em cães, sem qualquer método de exclusão, justamente com o objetivo de analisar a rotina do laboratório. Destes, os que apresentaram o trofozoíto da Babesia spp. na citologia dos esfregaços sanguíneos foram submetidos também à avaliação do resultado de seus exames em busca da identificação de um possível padrão entre as alterações hematológicas.

A metodologia adotada para a realização do hemograma foi de acordo com Jain, Feldman e Zinkl (2000). Assim, todas as amostras de sangue analisadas chegaram em um tubo de coleta com EDTA, do qual foram produzidos tubos capilares, para leitura da proteína total e hematócrito manual, e lâminas de esfregaço sanguíneo de cada amostra. Em seguida, as lâminas foram coradas pelo método panótico rápido e o sangue restante nos tubos foi passado em um analisador hematológico automatizado (pocH - 100iV Diff da Sysmex ${ }^{\circledR}$ ), de onde foram obtidos os valores de eritrócitos, hemoglobina, hematócrito, CHGM e VGM para o eritrograma e valores gerais de leucócitos para o leucograma, além da contagem de plaquetas. Por fim, as lâminas de esfregaço sanguíneo foram lidas em microscopia óptica em aumento de imersão (100x) para contagem diferencial de leucócitos, análise da morfologia celular, e eventuais achados hematológicos, dentre eles inclusões e presença de hematozoários, como a Babesia spp. (Figura 1).

Os valores referenciais utilizados para análise dos resultados laboratoriais foram divididos conforme a faixa etária dos pacientes. Dessa forma, os cães que tinham até três meses de idade, foram analisados seguindo os parâmetros empregados por Meyer e Harvey (2004), e, os exames dos cães acima desta faixa etária, segundo Jain et al. (2000). Os dados obtidos, por sua vez, foram organizados em tabelas e submetidos a avaliações estatísticas. De acordo com Pereira et al. (2018), a perspectiva matemática é de suma importância, já que além de permitir a previsão dos acontecimentos, também fornece ferramentas para melhor entendê-los.

Figura 1. Microscopia óptica de esfregaço sanguíneo em aumento de imersão (100x) com a presença de um trofozoíto de Babesia spp. no centro da imagem.

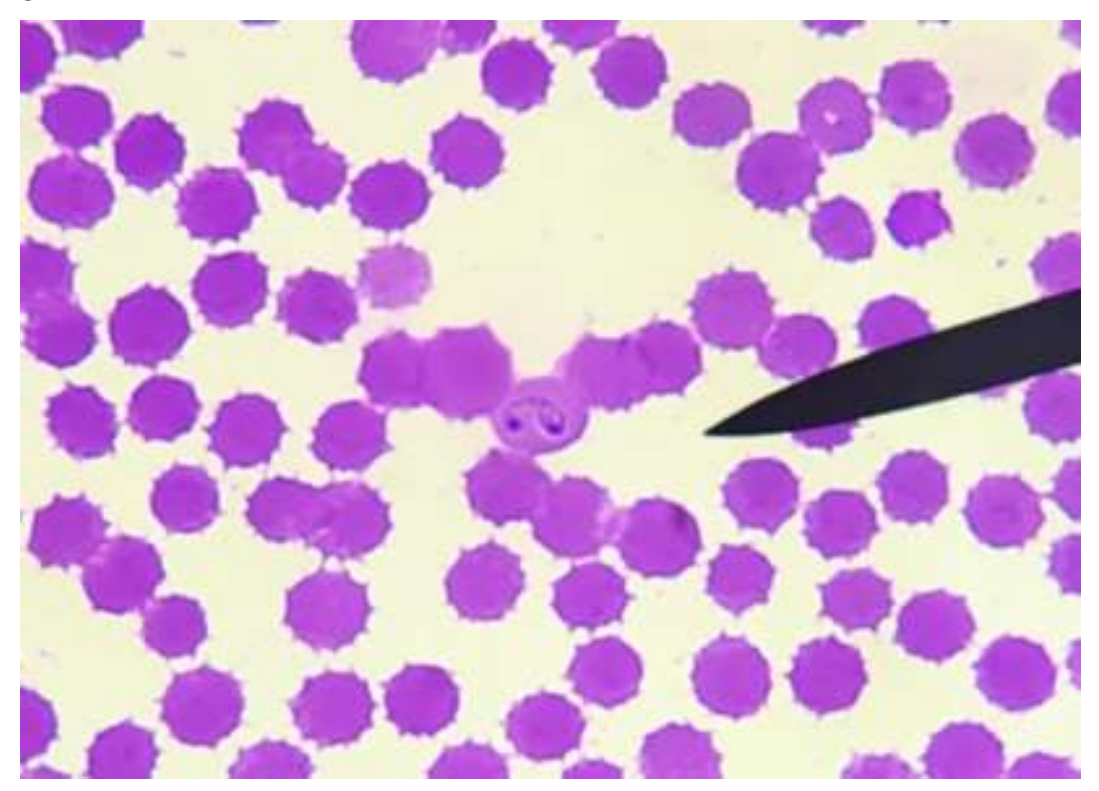

Fonte: LPCV - HOVET/UFMT. 


\section{Resultados e Discussão}

Dezesseis dos 5.333 hemogramas em cães foram considerados parasitados, pela presença do trofozoíto da Babesia spp. no exame microscópico direto, caracterizando a prevalência de $0,3 \%$ da hemoparasitose na rotina do LPCV do HOVETUFMT. O índice de parasitismo em lâmina foi inferior aos descritos por Miranda, Albernaz, Melo Junior e Machado (2008), e Santos et al. (2018), conforme disposto na Tabela 1. Não foi encontrado na literatura ocorrência inferior à observada no presente estudo.

Tabela 1. Estudos referentes a ocorrência de Babesia spp. por meio de citologia sanguínea em diferentes localidades do Brasil.

\begin{tabular}{llll}
\hline Local & Número de cães & $\begin{array}{l}\text { \% infectados por } \\
\text { Babesia spp. }\end{array}$ & Referência \\
\hline Campos dos Goytacases (RJ) & 2.031 & 1,47 & Miranda et al. (2008) \\
Campo Grande (MS) & 260 & 0,8 & Santos et al. (2018) \\
Cuiabá (MT) & 5.333 & 0,3 & Presente relato \\
\hline
\end{tabular}

Fonte: Arquivo pessoal.

Há diversos fatores importantes a serem considerados para a análise deste resultado, dentre eles, a amostra consideravelmente maior empregada, visto que por ser uma análise de ocorrência, espera-se que quanto maior o $n$ do estudo, menor seja o resultado obtido. Ainda mais, existe o fato de que os exames utilizados nesta pesquisa foram referentes a rotina do LPCV, sem seleção de animais por sintomatologia, sexo, raça ou idade. Com isto, vale ressaltar que o escopo da pesquisa de Santos et al. (2018), considerou apenas cães com a suspeita confirmada de hemoparasitoses, logo, é natural que sua ocorrência seja maior, já que foi relacionada a outras parasitoses sanguíneas, não necessariamente à rotina clínica do local.

Vale ressaltar que $75 \%$ dos exames em que a presença da Babesia spp. foi detectada, no presente relato, chegaram para análise com suspeita de outras doenças, sendo o diagnóstico firmado de forma esporádica. Isso evidencia que o esfregaço sanguíneo é uma ferramenta essencial para o reconhecimento da hemoparasitose, principalmente, pelo fato de a doença apresentar sintomatologia altamente inespecífica (Dias \& Ferreira, 2016).

Cerca de 75\% (12/16) dos achados de Babesia spp. se deu em cães com até 1 (um) ano de idade (Figura 1), resultado semelhante ao encontrado por Silva et al. (2012) e Castro (2020), que também observaram maior prevalência nessa faixa etária. Segundo Silva et al. (2012), isso pode se dar devido à idade ser um importante fator de resistência, sendo os filhotes, então, mais susceptíveis à contaminação. Apesar disso, há autores que descrevem o maior acometimento da doença em animais adultos (Trapp, Messick \& Vidotto, 2006; Guimarães et al., 2009), argumentando que tanto um maior tempo de exposição ao vetor quanto uma maior infestação aumentam a possibilidade de aquisição da babesiose. 
Figura 2. Gráfico referente à idade de acometimento de cães pela babesiose identificados no LPCV/HOVET-UFMT em 2019.

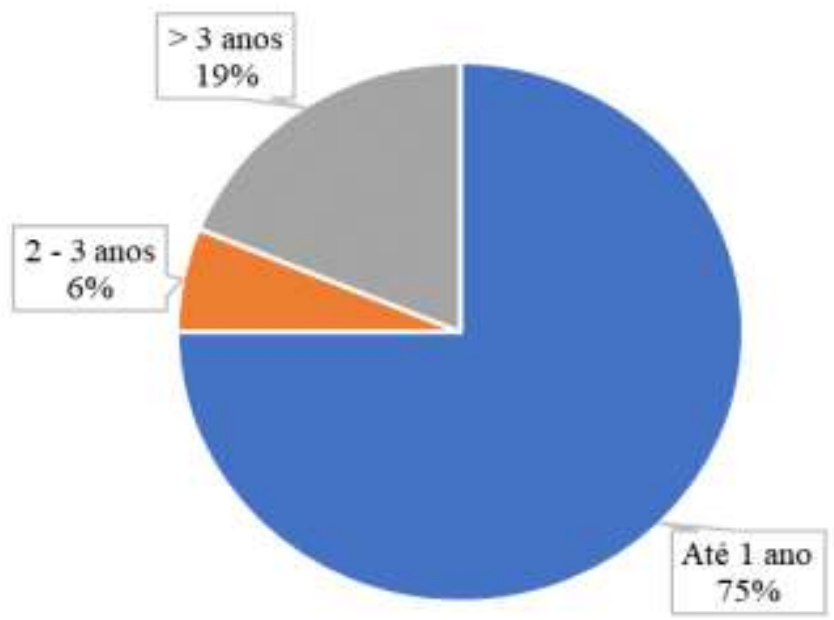

Fonte: Arquivo pessoal.

No eritrograma, foi observado anemia em $68,75 \%$ dos casos. Sabe-se que a anemia hemolítica grave é o achado mais comum da babesiose, apresentando risco de morte para cães jovens ou com alta carga parasitária (Thrall, Weiser, Robin \& Campbell, 2015). Segundo Irwin (2005) e Weiss e Wardrop (2010), a hemólise pode ocorrer de forma intra ou extravascular, sendo a resposta imunológica o mecanismo mais importante na patologia desta enfermidade, através da produção de anticorpos intra-eritrocitários. Ainda mais, 45,45\% deles apresentaram anemia normocítica normocrômica (Figura 3), como também encontrado por Fernandes, Nagayoshi e Barbosa (2013). Tal resultado é o esperado, começando com baixa intensidade nos primeiros dias após a infecção, posteriormente, evoluindo para uma anemia macrocítica hipocrômica e regenerativa à medida que a moléstia progride (Dias \& Ferreira, 2016).

Figura 3. Gráfico da análise dos valores obtidos do VGM e CHGM dos cães anêmicos positivos para Babesia spp. na microscopia direta no LPCV/HOVET-UFMT em 2019.

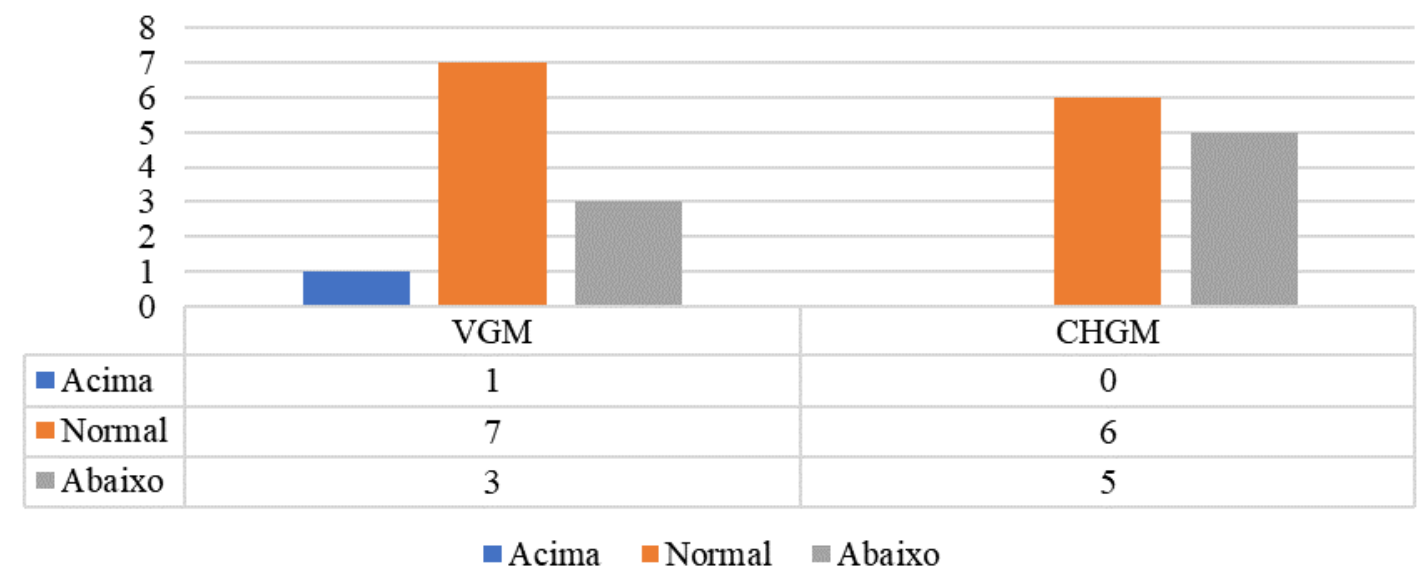

Fonte: Arquivo pessoal.

Segundo Moraes, Takahira, Golim e Baggio (2016), a trombocitopenia é uma das principais anormalidades hematológicas observadas na infecção por Babesia spp., consequentemente, a contagem total de plaquetas no presente estudo confirmou isto, apresentando a alteração em cerca de $87,5 \%$ dos casos confirmados. Comumente, a trombocitopenia é atribuída 
à ocorrência de CID, tendo como causas predisponentes hemólise, vasculite, acidose, hipóxia, dentre outras, sendo estas alterações clínicas comuns na babesiose (Campos et al., 2002).

Já quanto aos valores de PPT, 81,25\% apresentaram-se dentro dos referenciais, outros 12,5\% hiperproteinemia e 6,25\% hipoproteinemia. Segundo Furlanello, Fiorio, Caldin, Lubas e Solano-Gallego (2005), em sua forma mais severa, a Babesia canis, pode levar à redução dos níveis séricos de albumina, provavelmente, em decorrência de ser uma proteína de fase aguda negativa. Isso pôde ser visto no estudo realizado por Canuto et al. (2016), no qual cerca de 44,44\% dos animais apresentaram diminuição das PPT e somente 7,40\% hiperproteinemia.

De acordo com Vercammen, Deken e Maes (1997), as anormalidades leucocitárias observadas são variadas, podendo apresentar leucocitose ou leucopenia, neutrofilia ou neutropenia, linfocitose, eosinofilia e monocitose. No presente trabalho, foi observado leucopenia em $75 \%$ dos casos e em 18,75\% observou-se leucocitose, neutropenia em 50\%, neutrofilia em 12,5\%, eosinopenia em 68,75\%, linfopenia em 87,5\% e monocitose em 18,75\%, como pode ser visto na Figura 2.

A leucopenia verificada foi semelhante à encontrada por Fernandes et al. (2013) e Canuto et al. (2016), dessa forma, a alteração, juntamente com a neutropenia, pode ocorrer em detrimento da migração de neutrófilos para o tecido inflamado, excedendo sua liberação na medula (Stockham \& Scott, 2011). Ademais, sabe-se que a anemia não responsiva, associada a trombocitopenia e a leucopenia intensa, podem indicar hipoplasia medular, consequentemente, essas foram as principais desconformidades achadas nos exames.

Já a diminuição de eosinófilos encontrada é incomum em animais com babesiose, apesar de já ter sido relatada por Canuto et al. (2016). Vale ressaltar, que a eosinopenia detectada associada à linfopenia, pode estar relacionada à estresse, pelo aumento de cortisol endógeno, causado muitas vezes por dor. Por fim, o aumento de monócitos era esperado, pois reflete uma necessidade de macrófagos no sangue, ativando o sistema fagocitário mononuclear, muitas vezes em decorrência da hemólise intensa (Stockham \& Scott, 2011).

Figura 4. Gráfico de análise do leucograma dos cães positivos para Babesia spp. na microscopia direta no LPCV/HOVETUFMT em 2019.

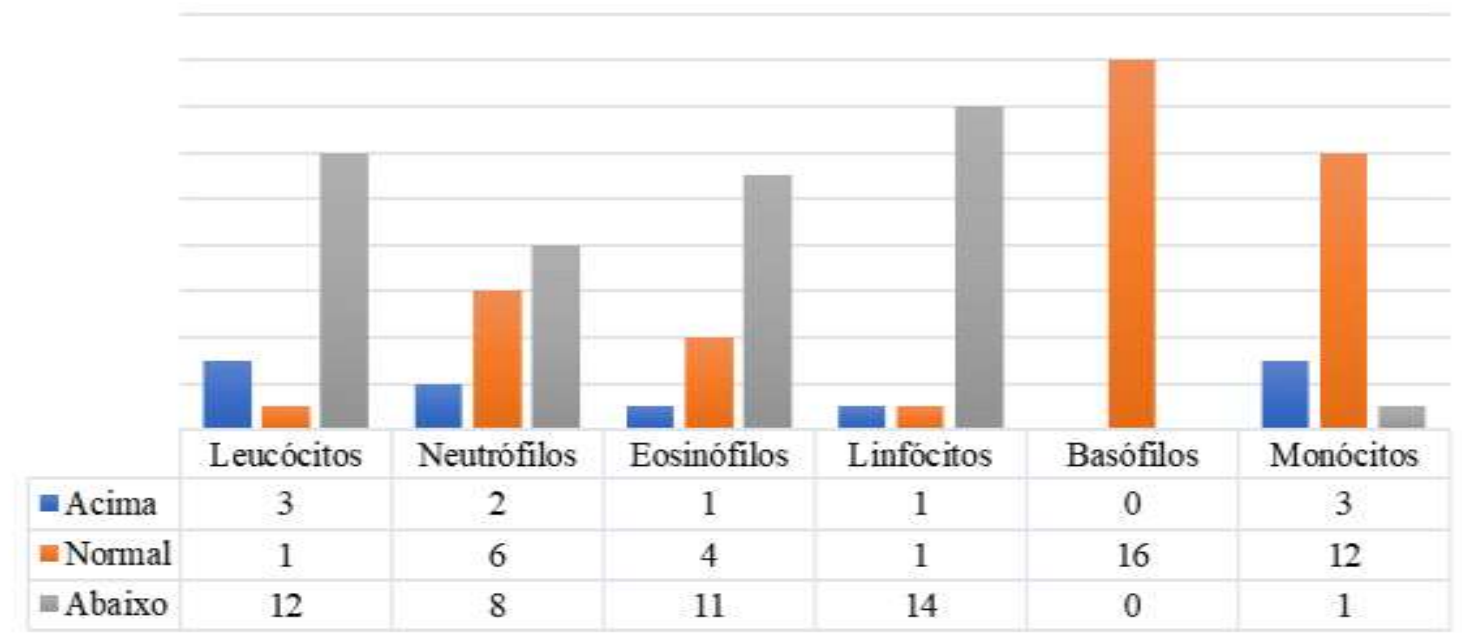

Fonte: Arquivo pessoal.

\section{Conclusão}

Neste estudo, verificou-se a ocorrência de $0,3 \%$ de babesiose em cães na rotina do LPCV do HOVET - UFMT, campus Cuiabá, durante o ano de 2019. Embora seja uma porcentagem baixa, 75\% dos animais foram diagnosticados de forma 
esporádica, sem sequer suspeita de hemoparasitose. Assim, nota-se a importância deste resultado como exame de triagem, já que por ser um teste altamente conclusivo, os cães que apresentam o trofozoíto da Babesia spp. no esfregaço evidentemente possuem a doença. Portanto, com o diagnóstico rápido, é possível proporcionar um protocolo de tratamento adequado e imediato.

Ainda mais, os principais achados hematológicos foram a anemia $(68,75 \%)$ normocítica normocrômica $(45,45 \%)$, trombocitopenia (87,5\%), leucopenia (75\%), eosinopenia (68,75\%), linfopenia (87,5\%) e monocitose (18,75\%). Frente a isso, é fundamental ressaltar a importância da citologia sanguínea como diagnóstico desta enfermidade, uma vez que mesmo em casos que exame microscópico direto resulte negativo, com o histórico clínico associado ao perfil hematológico, especialmente em animais jovens ou menores de um ano de idade, é possível validar a suspeita, obtendo a confirmação por meio de diagnósticos moleculares, aumentando as chances de fechamento de diagnóstico.

Por fim, para futuros trabalhos, é sugerida uma atenção especial aos valores de eosinófilos a fim de compreender melhor a alteração, seja ela seu aumento ou diminuição. Ademais, que busquem destacar a importância da técnica empregada, bem como do profissional que a realiza.

\section{Referências}

Andereg, P. I. \& Passos, L. M. F. (1999). Erliquiose canina: revisão. Clínica Veterinária, 4(18): 31-38.

Brandão, L. P. \& Hagiwara, M. K. (2002). Babesiose canina: revisão. Clínica Veterinária, 7(41): 50-59.

Campos, K. C. H., Santos, L. P., Takahira, R. K., Lopes, R. S., Silveira, V. F. \& Mattoso, C. R. S. (2002). Incidência de trombocitopenia em cães naturalmente infectados por Babesia canis. In Anais $6^{a}$ Mostra Científica da Faculdade de Medicina Veterinária e Zootecnia - UNESP (56), Botucatu. Botucatu: FMVZUNESP.

Canuto, F. J. C., Matias, A. L. M. \& Cortez A. A. (2016). Achados hematológicos de cães positivos para babesiose em Fortaleza, Ceará. In IV Congresso Estudantil de Medicina Veterinária da UECE 26 (10-12), Fortaleza. Fortaleza: Ciência Animal.

Castro, V. V., Ayres, E. C. B. S., Canei, D. H., Pereira, M. E., Souza, V. R. F., Chitarra, C. S., Dutra, V., Nakazato, L. \& Almeida, A. B. P. F. (2020). Molecular prevalence and factors associated with Babesia vogeli infection in dogs in the Cerrado Mato-Grossense region of Brazil. Ciência Rural, 50(2). https://dx.doi.org/10.1590/0103-8478cr20190389.

Day, M. J. (2011). One health: the importance of companion animal vector-borne diseases. Parasites \& vectors, 4(1): 49.

Dias, V. A. C. M. \& Ferreira, F. L. A. (2016). Babesiose canina: Revisão. Pubvet, 10: 873-945.

Duarte, S. C., Louly, C. C. B., Silveira Neto, O. J., Romanowski, T. N. A., Lino Junior, R. S. \& Linhares, G. F. C. (2008). Parasitologic and molecular diagnosis of canine babesiosis in Goiânia, Brazil. Revista de Patologia Tropical / Journal of Tropical Pathology, $37(3)$ : 229-236. https://doi.org/10.5216/rpt.v37i3.5064.

Fernandes, L. L., Nagayoshi, B. A. \& Barbosa, T. S. (2013). Hematologia dos cães com babesiose atendidos no Hospital Veterinário da Universidade de Marília - UNIMAR. Revista Unimar Ciências, 22(1-2): 49-52.

Furlanello, T., Fiorio, F., Caldin, M., Lubas, G. \& Solano-Gallego, L. (2005). Clinicopathological findings in naturally occurring cases of babesiosis caused by large form Babesia from dogs of northeastern Italy. Veterinary Parasitology, 134(1-2): 77-85. https://doi.org/10.1016/j.vetpar.2005.07.016.

Guimarães, A. M., Rocha, C. M. B. M., Oliveira, T. M. F. S., Rosado, I. R., Morais, L. G. \& Santos, R. R. D. (2009). Fatores associados à soropositividade para Babesia, Toxoplasma, Neospora e Leishmania em cães atendidos em nove clínicas veterinárias do município de Lavras, MG. Revista Brasileira de Parasitologia Veterinária, 18(1): 40-53. https://doi.org/10.4322/rbpv.018e1009.

Irwin, P. J. (2005). Babesiosis and cytauxzoonosis. In Shaw, S. E., \& Day, M. J. (Eds), Arthropod-borne infectious diseases of the dog and cat. Manson Publishing, Barcelona, pp. 63-77.

Jain N. C., Feldman, B. F., \& Zinkl, J. G. (2000). Schalm's veterinary hematology (5a ed). Philadelphia: Lea \& Febiger.

Meyer, D. J. \& Harvey, J. W. (2004). Veterinary laboratory medicine: interpretation \& diagnosis (2a ed). Philadelphia: Sauders, 2004.

Miranda, F. J. B., Albernaz, A. P., Melo Junior, O. A. \& Machado, J. A. (2008). Frequência de cães infectados por Babesia spp. em Campos dos Goytacases, RJ. Ciência Animal Brasileira, 9(1): 238-241.

Moraes, L. F., Takahira, R. K., Golim, M. A. \& Baggio, M. S. (2016). Avaliação das alterações hemostáticas e do risco tromboembólico em cães com AHIM. Pesquisa Veterinária Brasileira, 36(5): 405-411. https://doi.org/10.1590/S0100-736X2016000500009.

Pereira, A. S., Shitsuka, D. M., Parreira, F. J., \& Shitsuka, R. (2018). Metodologia da Pesquisa Científica. UAB/NTE/UFSM. https://repositorio.ufsm.br/bitstream/handle/1/15824/Lic_Computacao_Metodologia-Pesquisa-Cientifica.pdf?sequence=1 . 
Research, Society and Development, v. 10, n. 2, e1810211907, 2021

(CC BY 4.0) | ISSN 2525-3409 | DOI: http://dx.doi.org/10.33448/rsd-v10i2.11907

Santos, C. M., Conte, F. O., Tonial, A. L., Bairros, A. A., Aquino, D. R. R. R. A. \& Favacho, A. R. M. (2018). Ocorrência de hemoparasitose em cães atendidos em hospital veterinário de Campo Grande, estado do Mato Grosso do Sul, Brasil. Brazilian Journal of Animal and Environmental Research, 1(1): 236-243.

Stockham, S. L. \& Scott, M. A. (2011). Fundamentos da patologia clínica veterinária (2a ed.). Guanabara Koogan.

Serra-Freire, N. M. (2014). Caso Índice de babesiose humana no Rio de Janeiro, Brasil. Revista UNIABEU, 7(15): 76-85.

Silva, A. B., Costa, A. P., Sá, J. C., Costa, F. B., Santos, A. C. G. \& Guerra, R. M. S. N. C. (2012). Detecção molecular de Babesia canis vogeli em cães e em Rhipcephalus sanguineus na mesorregião do oeste maranhense, nordeste brasileiro. Ciência Animal Brasileira, 13(3): 388-395.

Thrall, M. A., Weiser, G., Robin, W. A. \& Campbell, T. W. (2015). Hematologia e Bioquímica Veterinária (2a ed). Roca.

Trapp. S. M., Messick, J. B. \& Vidotto, O. (2006). Babesia gibsoni genotype Asia in dogs from Brazil. Veterinary Parasitology, 141(1-2): 177-180. https://doi.org/10.1016/j.vetpar.2006.04.036.

Vercammen, F., Deken, R. \& Maes, L. (1995) Clinical and serological observations on experimental infections with Babesia canis and its diagnosis using the IFAT. Parasite, 2(4): 407-410.

Vercammen, F., Deken, R. \& Maes, L. (1997). Duration of protective immunity in experimental canine babesiosis after homologous and heterologous challenge. Veterinary Parasitology, 68: 51-55.

Weiss, D. \& Wardrop, K. (2010). Schalm's Veterinary Hematology (6a ed). Wiley-Blackwell. 